\title{
La congrégation des Tertiaires capucins et la réception des modèles internationaux de prise en charge des jeunes délinquants (Espagne, 1904-1936)
}

The congregation of Capuchin Tertiary Sisters and receiving international models for caring for young delinquents (Spain, 1904-1936)

La orden de los Terciarios Capuchinos et la recepción de los modelos internacionales de reeducación de los jóvenes delincuentes (España, 1904-1936)

\section{Amélie Nuq}

\section{OpenEdition}

\section{Journals}

Édition électronique

URL : https://journals.openedition.org/histoire-education/2850

DOI : 10.4000/histoire-education.2850

ISSN : 2102-5452

Éditeur

ENS Éditions

Édition imprimée

Date de publication : 31 août 2014

Pagination : 157-174

ISBN : 978-2-84788-649-8

ISSN : 0221-6280

\section{Référence électronique}

Amélie Nuq, « La congrégation des Tertiaires capucins et la réception des modèles internationaux de prise en charge des jeunes délinquants (Espagne, 1904-1936) ». Histoire de l'éducation [En ligne], 140-141 | 2014, mis en ligne le 31 août 2016, consulté le 20 mai 2021. URL : http:// journals.openedition.org/histoire-education/2850; DOI : https://doi.org/10.4000/histoire-education. 2850 


\section{La congrégation des Tertiaires capucins et la réception des modèles internationaux de prise en charge des jeunes délinquants (Espagne, 1904-1936)}

Amélie Nuq

La congrégation espagnole des Tertiaires capucins de Notre-Dame des douleurs est fondée en 1889 par le père Luis Amigó y Ferrer : spécifiquement destinée à l'éducation correctionnelle et à la moralisation des jeunes délinquants, elle prend la tête de la quasi-totalité des maisons de redressement espagnoles à mesure que celles-ci sont créées. Mais rapidement, la formation insuffisante de ses membres est pointée du doigt. C'est la raison pour laquelle la congrégation organise, au début du $\mathrm{XX}^{\mathrm{e}}$ siècle, une série de voyages destinés à observer le fonctionnement d'institutions rééducatives en Allemagne, en Angleterre, en Belgique, en France, en Italie, aux Pays-Bas et en Suisse. De ces observations, les Tertiaires capucins rapportent un certain nombre d'éléments dans la perspective de les transposer dans leur propre système de prise en charge de la jeunesse "délinquante". Ce processus peut être saisi par l'analyse du récit de ces voyages dans les périodiques émanant de la congrégation elle-même ${ }^{2}$,

1 Le terme de mineurs délinquants est le plus couramment utilisé dans la littérature de l'époque même si les individus concernés n'ont pas toujours commis un délit à proprement parler; pour notre part, nous retiendrons le terme de jeunesse déviante, qui permet d'englober l'ensemble des mineurs pris en charge.

2 Il s'agit des revues Adolescens, Surge! (1931-1936) et ¡Surgam...! (1949-2005). La première est accessible sur le site internet de la congrégation religieuse (<http://www.amigonianos.org >); la 
seule source disponible, les archives capucines, conservées à Rome, n'étant pas accessibles ${ }^{3}$.

Il est désormais admis que les circulations internationales ont joué un rôle important dans l'élaboration des politiques sociales à partir de la fin du $\mathrm{XIX}^{\mathrm{e}}$ siècle ${ }^{4}$. Plus précisément, les voyages d'études sont l'une des manifestations de la socialisation internationale qui émerge progressivement dans le domaine social ${ }^{5}$. Les excursions en terre étrangère menées par les Tertiaires capucins sont encore peu connues, même en Espagne; de façon générale, la production scientifique relative à la jeunesse déviante y est limitée alors que ce champ est dynamique en France. Les ouvrages publiés par les historiens officiels des Tertiaires capucins, qui sont eux-mêmes des religieux et ont à ce titre eu accès aux archives de la congrégation, ont une visée clairement hagiographique ${ }^{6}$. La présente contribution s'inscrit pour sa part dans un mouvement plus large qui s'intéresse à l'internationalisation dans le domaine de la protection de l'enfance ${ }^{7}$ et dans celui de l'éducation ${ }^{8}$. Ce faisant, elle tentera de démêler l'écheveau des connexions entretenues par les Tertiaires capucins avec l'étranger pour mettre en évidence d'éventuels "régimes circulatoires" dans le domaine de la prise en charge de la jeunesse déviante. Nous éclairerons ainsi un angle mort de l'historiographie : le cas de la péninsule ibérique n'est pas abordé dans les travaux soulignant la dimension transnationale de l'histoire de la protection de l'enfance, qu'il s'agisse d'études comparatives ${ }^{9}$ ou d'ouvrages portant sur les

seconde est consultable à la Bibliothèque nationale espagnole, à Madrid. Enfin, les textes pédagogiques écrits par des Tertiaires capucins ont fait l'objet d'une publication en 24 volumes; voir par exemple Lorenzo María de Alquería de la Condesa, Obras, Madrid, Congregación Religiosos Terciarios Capuchinos de Nuestra Señora de los Dolores, D.L., 1993.

3 Malgré nos demandes réitérées, nous n'avons jamais obtenu le droit de consulter cette documentation.

4 Sandrine Kott, "Une "communauté épistémique” du social? Experts de l'OIT et internationalisation des politiques sociales dans l'entre-deux-guerres", Genèses, n71, 2008, p. 41.

5 Pierre-Yves Saunier, "Les régimes circulatoires du domaine social 1800-1940 : projets et ingénierie de la convergence et de la différence", Genèses, $n^{\circ} 71,2008$ p. 9; idem, "Circulations, connexions et espaces transnationaux", Genèses, n57, 2004, p. 113.

6 Tomás Roca Chust, Historia de la Congregación de Religiosos Terciarios Capuchinos de Nuestra Señora de los Dolores, Madrid, Gráficas Lersi, 1968-2011, 8 vol. ; Juan Antonio Vives Aguilella, Manual de historia de la Congregación Terciarios Capuchinos (1889-2002), Rome, J. Vives, 2005.

7 Voir par exemple Joëlle Droux, «L'internationalisation de la protection de l'enfance : acteurs, concurrences et projets transnationaux (1900-1925)", Critique internationale, n52, 2011, p. 17-33.

8 En 2012 s'est par exemple tenu, à Genève, le 34 congrès de l'International Standing Conference for the History of Education, qui s'est interrogée sur "L'internationalisation dans le champ éducatif (18 $-20^{\mathrm{e}}$ siècles)".

9 David Niget, La naissance du tribunal pour enfants. Une comparaison France-Québec (1912-1945), Rennes, Presses universitaires de Rennes, 2009. Pour un état synthétique de la question, voir Samuel 
circulations et les transferts ayant eu lieu à la fin du XIX ${ }^{\mathrm{e}}$ siècle et au début du $\mathrm{XX}^{\mathrm{e}}$ siècle $^{10}$. Il s'agira alors de se demander si l'Espagne constitue un exemple supplémentaire qui corrobore la "dimension fondamentalement transnationale du "domaine social” " ? ${ }^{11}$ Enfin, la série de voyages d'études organisée par les Tertiaires capucins est un exemple concret, vu d'en bas, de la circulation des modèles de protection et d'encadrement de la jeunesse ${ }^{12}$. Il ne s'agit pas seulement de descendre à une autre échelle mais de faire en quelque sorte un pas de côté pour regarder autrement et repérer des connexions qui étaient jusque-là inaperçues ${ }^{13}$.

Nous présenterons d'abord le rôle joué par les Tertiaires capucins dans la mise en place des maisons de redressement en Espagne. Nous décrirons ensuite le déroulement des voyages d'étude réalisés pendant le premier tiers du $\mathrm{XX}^{\mathrm{e}}$ siècle, avant de nous demander si ceuX-ci aboutissent effectivement à un transfert des méthodes et des pratiques observées à l'étranger.

\section{La place des Tertiaires capucins dans la genèse des maisons de redressement espagnoles, entre inexpérience et quasi-monopole éducatif}

\section{L'École de Santa Rita, symbole du retard espagnol par rapport aux autres pays occidentaux}

$\mathrm{Au} \mathrm{XIX}^{\mathrm{e}}$ siècle, le retard accumulé par l'Espagne dans le domaine de la prise en charge de la jeunesse déviante est considérable. En France, en Belgique et aux Pays-Bas, les premiers établissements spécifiquement destinés aux jeunes

Boussion, "L'Association internationale des éducateurs de jeunes inadaptés (AIEJI) et la fabrique de l'éducateur spécialisé par delà les frontières (1951-1963) ", Paedagogica Historica. International Journal of the History of Education, vol. 50, n 1-2, février-avril 2014, p. 229-243.

10 Voir notamment Marie-Sylvie Dupont-Bouchat, Éric Pierre (dir.), Enfants et justice au XIXe siècle. Essai d'histoire comparée de la protection de l'enfance 1820-1914, France, Belgique, Pays-Bas, Canada, Paris, Presses universitaires de France, 2001.

11 Pierre-Yves Saunier, "Les régimes circulatoires...", art. cit., p. 13.

12 Selon Sven Beckert et Matthew Connelly, cette dimension empirique manque justement aux études d'histoire transnationale. C.A. Bayly, Sven Beckert, Matthew Connelly, Isabel Hofmeyr, Wendy Kozol, Patricia See, "AHR conversation: On transnational history", The American Historical Review, vol. 111, n5, décembre 2006, p. 1446-1447.

13 Sanjay Subrahmanyam, Explorations in Connected History. From the Tagus to the Ganges, Oxford, Oxford University Press, 2005, p. 11. Cité par Caroline Douki, Philippe Minard, "Histoire globale, histoires connectées : un changement d'échelle historiographique?", Revue d'histoire moderne et contemporaine, $\mathrm{n}^{\circ} 54-4$ bis, 2007, p. 20. 
délinquants se sont en effet multipliés au cours des années 1830 et 1840, tandis que les années 1870-1890 donnaient lieu à une remise en cause des établissements correctionnels. Dans la péninsule, il faut attendre 1883 pour que soit décidée la création d'un établissement de redressement et de correction, l'école de réforme et asile de correction paternelle de Santa Rita ${ }^{14}$. En 1890, les Tertiaires capucins prennent la direction de cette institution madrilène destinée à "recueillir et éduquer les jeunes vicieux et vagabonds ainsi que les fils de famille méritant une correction ${ }^{15}$. C'est le premier établissement de ce type quand, à la même date, la France compte déjà vingt-deux institutions privées ${ }^{16}$. L'école de Santa Rita est régie par une assemblée de patronage : cet appel au secteur privé religieux n'est pas spécifique à l'Espagne. Joëlle Droux a montré que, dans l'ensemble des pays occidentaux, les dispositifs de protection de la jeunesse mis en place à la fin du XIX et au début du XX $\mathrm{XX}^{\mathrm{e}}$ siècle faisaient des œuvres privées les principaux agents d'exécution des mesures civiles ou pénales ${ }^{17}$. Mais la genèse tardive de Santa Rita s'inscrit dans le processus particulier de construction de l'État espagnol, "utopie réactionnaire" au long cours se caractérisant par un manque de moyens structurel, par le poids des élites traditionnelles et par la place importante de l'Église catholique ${ }^{18}$.

L'établissement madrilène ne pouvant fonctionner sans personnel, l'assemblée de patronage tourne son regard vers le secteur privé catholique : seuls des religieux auraient la vocation suffisante et l'esprit assez désintéressé pour assurer la direction de l'établissement ${ }^{19}$. L'éducation religieuse doit d'ailleurs être au fondement de la correction et de la réforme des adolescents : chez

14 Ley autorizando á la Junta de Patronos, y en su representación a la Comisión ejecutiva que venía entendiendo en el proyecto de establecer una penitenciaría de jóvenes, para fundar un asilo de corrección paternal y una escuela de reforma en donde reciban educación correccional los jóvenes menores de 18 años, Gaceta de Madrid, nº, 6 janvier 1883. Le texte s'inspire probablement du modèle des "écoles de réforme" fondées en 1848 en Belgique et repris ensuite aux Pays-Bas.

15 Pour une étude détaillée de la genèse et du fonctionnement de Santa Rita, voir Amélie Nuq, "La première maison de redressement espagnole, l'école de réforme et l'asile de correction paternelle de Santa Rita (1883-1936)", in Xavier Huetz de Lemps, Jean-Philippe Luis (dir.), Sortir du labyrinthe. Études d'histoire contemporaine de l'Espagne, Madrid, Casa de Velázquez, 2012, p. 403-430.

16 Marie-Sylvie Dupont-Bouchat, Éric Pierre (dir.), Enfants et justice au XIX ${ }^{e}$ siècle..., op. cit., p. 298.

17 Joëlle Droux, "L'internationalisation de la protection de l'enfance...", art. cit., p. 20.

18 Jean-Philippe Luis a qualifié d'"utopie réactionnaire" la politique menée par Ferdinand VII de 1823 à 1833, qui contribue à créer un État de transition entre l'Ancien Régime et la société libérale. Jean-Philippe Luis, L'utopie réactionnaire. Épuration et modernisation de l'État dans l'Espagne de la fin de l'Ancien Régime (1823-1831), Madrid, Casa de Velázquez, 2002.

19 Manuel Cossío y Gómez-Acebo, Proyecto de organización de las Instituciones tutelares de la Infancia abandonada: memoria, Madrid, Real Casa, 1907, p. 102-103. 
ces philanthropes qui sont à la fois des hommes politiques, des savants, des penseurs et des gestionnaires, présents aussi bien sur les bancs du Congrès des députés que dans les associations de protection de l'enfance, cette idée se combine à une vision paternaliste et méprisante des classes populaires ${ }^{20}$. Les deux délégués espagnols au Congrès pénitentiaire de Rome (1885), Francisco Silvela et Francisco Lastres, profitent de leur présence en Italie pour visiter l'asile tenu par Don Bosco mais les Salésiens refusent de prendre en charge l'école de Santa Rita. En 1888, le problème lancinant du personnel n'étant toujours pas réglé, le pape Léon XIII conseille à Lastres de se tourner vers la congrégation des Tertiaires capucins, qui vient d'être créée par Luis Amigó y Ferrer.

Né en 1854 à Masamagrell, près de Valence, Luis Amigó y Ferrer a fait des études d'humanités et de philosophie au Séminaire de Valence ${ }^{21}$. En 1874, il prend l'habit capucin et est ordonné prêtre. C'est en visitant une prison à Santander qu'il conçoit l'idée de fonder un ordre religieux s'investissant dans la réhabilitation des délinquants, et tout particulièrement dans celle des mineurs. En 1881, il commence à rédiger les statuts qui deviennent, en 1885, les Constitutions des Tertiaires capucines de la Sainte Famille (Terciarias Capuchinas de la Sagrada Familia) et des Tertiaires capucins de Notre-Dame des Douleurs (Terciarios Capuchinos de Nuestra Señora de los Dolores). Ces deux ordres religieux "frère" et "sœur" sont spécifiquement destinés à l'éducation correctionnelle, à la moralisation et l'enseignement des sciences et des arts dans les établissements de redressement, que ceux-ci soient publics ou privés. Ils ont ainsi pour but d'occuper totalement l'espace de l'éducation correctionnelle, tout en respectant la stricte ségrégation selon le sexe qui régit tout autant ce champ que le cadre religieux à cette époque. En 1890, les Tertiaires capucins acceptent de prendre la direction de l'école de Santa Rita. En 1895, une ordonnance royale approuve l'installation de l'ordre en Espagne, marquant le début d'une expérience qui va durer plus de 130 ans et voir la congrégation prendre la tête de la quasi-totalité des maisons de redressement espagnoles.

\section{L'extension rapide du domaine capucin : modalités et critiques}

$\mathrm{Au}$ début du XX $\mathrm{XX}^{\mathrm{e}}$ siècle, le retard accumulé par l'Espagne revient à nouveau

20 Cette génération de nouveaux philanthropes, à cheval sur les secteurs public et privé, se développe au même moment dans les autres pays européens. Marie-Sylvie Dupont-Bouchat, Éric Pierre (dir.), Enfants et justice au XIX ${ }^{e}$ siècle..., op. cit., p. 20.

21 On pourra consulter Luis Amigó y Ferrer, Autobiografia, Valence, Terciarios Capuchinos, 1982. 
comme un leitmotiv dans les ouvrages des réformateurs sociaux, qui citent les exemples emblématiques de Red Hill en Angleterre, d'Alkmaar en Hollande, de Ruiselede en Belgique et de Mettray en France ${ }^{22}$. C'est à cette période que se développe un mouvement international en faveur de la protection de l'enfance, né dans le contexte relativement euphorique de prospérité économique ${ }^{23}$. Finalement, en 1918, comme d'autres pays occidentaux, l'Espagne commence à suivre l'exemple des États-Unis, où les enfants ne sont plus envoyés en prison, et institue des tribunaux pour enfants ${ }^{24}$. Notons que c'est la loi belge de 1912 qui influence le plus nettement le texte promulgué en Espagne en $1918^{25}$. L'idée fondamentale est que le mineur est différent de l'adulte et qu'au lieu de le punir, on doit plutôt le bien traiter, le rééduquer, le transformer. Les mesures éducatives que prescrit le tribunal pour enfants sont appliquées dans des établissements créés à cet effet, les reformatorios, qui sont le plus souvent tenus par des congrégations religieuses. En effet, le schéma qui a prévalu lors de la création de Santa Rita est, dans les grandes lignes, reproduit : l'État préfère déléguer la prise en charge de la déviance juvénile au secteur privé, les Tertiaires capucins s'engouffrant dans la brèche. Cette mainmise sur les institutions de redressement est à relier à l'action menée par l'épiscopat espagnol de la Restauration (1875-1930) qui, par le biais de congrégations religieuses créées ad hoc, impose son hégémonie sur le secteur éducatif ${ }^{26}$.

Cependant, à partir des années 1910, le monopole catholique acquis sur les établissements de redressement fait l'objet de critiques croissantes, qui n'émanent désormais plus de la seule presse républicaine anticléricale. Un ancien pensionnaire de Santa Rita, Abraham Polanco, lance par exemple

22 José Soler y Labernia, Nuestras cárceles, presidios y casas de corrección, Madrid, G. L. Del Horno, 1906, p. 8 et 115 .

23 Marie-Sylvie Dupont-Bouchat, "Le mouvement international en faveur de la protection de l'enfance (1880-1914)", Revue d'histoire de l'enfance "irrégulière", n5, 2003, p. 207-235.

24 Real decreto disponiendo se publiquen en este periódico oficial los artículos que forman la ley sobre Organización y atribuciones de los Tribunales para niños, y que se dé cuenta de la misma a las Cortes, Gaceta de Madrid, n³31, 27 novembre 1918. L'adoption de la loi Montero Ríos semble replacer l'Espagne dans une chronologie et un mouvement internationaux. Pourtant, cette synchronie est trompeuse : la lente dispersion des juridictions sur le territoire espagnol (il faudra attendre jusqu'en 1952 pour que chaque province dispose effectivement d'un tribunal pour mineurs) montre que la volonté politique et les moyens alloués sont insuffisants.

25 Dans les deux pays, on institue une juridiction spécialisée et un juge unique, par ailleurs assisté d'un agent de probation.

26 Manuel Puelles Benítez, Educación e ideología en la España contemporánea, Madrid, Tecnos, 1999, p. 248. 
une véritable campagne contre l'établissement, dénonçant "le fanatisme des Tertiaires capucins et leurs méthodes antiscientifiques ${ }^{27}$. Il affirme que cette "cléricaille" enferme les adolescents turbulents dans une prison indigne et honteuse. L'adage "maisons de correction, maisons de corruption" ne se serait jamais autant vérifié que dans l'établissement madrilène, "vivier de dégénérés " où l'homosexualité de certains frères corrompt les pensionnaires. Les critiques émanent parfois du personnel des juridictions lui-même ${ }^{28}$. Primitivo Requena, employé du tribunal pour mineurs de Madrid de 1925 à 1928, dénonce le fait que les religieux ne disposent d'aucune connaissance en psychologie, en pédagogie ou en droit ${ }^{29}$. À ces critiques s'ajoutent celles des esprits les plus progressistes du champ éducatif, membres de l'Institution libre d'enseignement (ILE). Cette organisation, créée en 1876, est attachée aux principes de liberté, de laïcité et d'indépendance de la science. Ses membres, convaincus que l'enfant est un "projet d'homme" qui doit être préservé des luttes idéologiques secouant la société, élèvent la neutralité religieuse à l'état de paradigme ${ }^{30}$. Dans le domaine de la prise en charge de la déviance juvénile, l'ILE accuse les religieux d'avoir une formation scientifique insuffisante, de fomenter l'hypocrisie, de pratiquer un faux paternalisme et d'employer des méthodes répressives. De fait, lorsque les Tertiaires capucins avaient accepté la direction de Santa Rita en 1890, ils étaient conscients de ne pas être suffisamment préparés et formés ${ }^{31}$.

\section{Les voyages d'étude à l'étranger, un moyen de structurer une congrégation religieuse encore jeune}

\section{Former des religieux capables d'éduquer en "servant Dieu grâce à la technique" 32}

En 1906, alors que la congrégation est fondée depuis plus de vingt ans déjà, le ministre général des Tertiaires capucins emploie encore le terme de "congrégation

27 Abraham Polanco, El correccional de Santa Rita: dos años entre sus muros: conferencias, notas, documentos y comentarios, Valladolid, Vd ${ }^{a}$ de Montero, 1914.

28 El País, 19 avril 1916.

29 Primitivo Requena, ¡Fracaso! El tribunal tutelar y el reformatorio de menores de Madrid, Madrid, Argis, 1932.

30 Manuel Puelles Benítez, Educación e ideología..., op. cit., p. 240-242.

31 Domingo de Guzmán, María de Alboraya, La Escuela de reforma de Santa Rita, situada en Carabanchel bajo, Madrid, Madrid, Hijos de M.G. Hernández,1906.

32 Tomás Roca Chust, Historia de la Congregación..., op. cit., p. 350. 
naissante". Il reconnaît implicitement le bien-fondé de certaines accusations : les novices ne sont pas assez soutenus et les études qu'ils sont censés suivre ne se passent pas bien, d'où un fonctionnement "anémique "33. Les témoignages d'anciens pensionnaires soulignent par ailleurs la division profonde qui existe entre les prêtres (sacerdotes) et les simples frères (hermanos) ${ }^{34}$. Les premiers, qui ont déjà prêté leurs vœux, constituent en quelque sorte l'aristocratie du personnel; les seconds, dotés d'un faible niveau d'instruction, appartiennent aux échelons inférieurs de la hiérarchie et accomplissent les tâches obscures du quotidien. Un ancien pensionnaire affirme par exemple que lorsqu'il a séjourné à Santa Rita, aucun religieux n'avait de diplôme. Il est en effet plausible qu'une congrégation aussi récente, aussi peu connue et qui a besoin de s'affirmer, ne soit pas très exigeante vis-à-vis du niveau des personnes qu'elle emploie. Par ailleurs, le recrutement de la congrégation valencienne est alors plutôt local et constitue un moyen, pour des familles modestes et peu éduquées, d'échapper à une vie de labeur aux champs ${ }^{35}$. L'ordre a d'autant plus besoin d'attirer de nouveaux membres que certains novices ont la vocation vacillante et que beaucoup quittent la congrégation ${ }^{36}$.

La faiblesse du niveau de formation des membres de la congrégation vient du fait que, jusque dans les années 1930, les moyens existants sont très limités. Les religieux peuvent assister à des stages (cursillos) organisés pour "transmettre aux nouvelles générations les méthodes pédagogiques amigoniennes ${ }^{37}$. Le centre basque d'Amurrio, l'une des premières maisons de redressement confiées aux Tertiaires capucins, accueille des sessions de formation estivales. Mais en dehors de ces initiatives ponctuelles, limitées et erratiques, il n'existe aucune structure éducative publique et centralisée qui forme le personnel exerçant dans les institutions correctives. La mise en place d'un tel centre est d'ailleurs une revendication récurrente de l'Institution libre d'enseignement. Selon l'historien officiel de la congrégation, Tomás Roca Chust, les supérieurs de l'ordre

33 Lettre adressée le 19 juillet 1906 par José María de Sedavi à Antonio Maura, président de l'assemblée de patronage de Santa Rita (Archives de la Fundación Antonio Maura, désormais FAM, 463/1).

34 L'écrivain franco-espagnol Michel del Castillo dresse le même constat à propos des années 1940, au sujet de l'Asilo Durán et d'une autre congrégation religieuse, celle de San Pedro Ad Víncula (entretien réalisé en juin 2010).

35 Miguel Mora Requeno, Los impunes. Historia del correccional de Santa Rita, Madrid, La cartelera artística, 1926, p.39 et 135-136.

36 Lettre d'Alacuás à Maura, 10 janvier 1920 (FAM, 463/13).

37 Tomás Roca Chust, Historia de la Congregación..., op. cit., p. 240. 
prennent en effet conscience du fait que "le devoir éducatif entraîne d'énormes difficultés et que le bon esprit religieux et le sens commun, si important chez le religieux éducateur, ne sont pas suffisants ${ }^{38}$.

\section{Aller chercher à l'étranger la réponse à des problèmes internes à la congrégation}

Les voyages d'étude apparaissent alors comme un moyen de pallier le manque de spécialisation et la formation insuffisante des Tertiaires capucins. Pendant le premier tiers du XX $\mathrm{XX}^{\mathrm{e}}$ siècle, plusieurs Tertiaires capucins vont ainsi observer le fonctionnement de plusieurs institutions rééducatives en France, en Angleterre, en Hollande, en Allemagne et surtout en Belgique. Le contexte international est alors favorable aux circulations d'expériences ${ }^{39}$. Depuis la fin du XIX ${ }^{\text {e }}$ siècle en effet, des acteurs divers (administrateurs, professeurs, industriels, syndicalistes, etc.) se retrouvent au sein de nébuleuses réformatrices qui jouent un rôle crucial dans la circulation des idées en matière sociale, comme dans la constitution et la diffusion de solutions "modèles $"^{40}$. Enfin, l'Etat espagnol encourage ces voyages, en prenant en charge les frais des déplacements à l'étranger organisés en 1909 : après avoir confié aux Tertiaires capucins l'organisation des maisons de redressement, il leur délègue donc une fonction d'observation internationale dans ce domaine.

Les récits de voyage montrent que les Tertiaires capucins sont avides d'apprendre : les visiteurs espagnols prennent des notes, relèvent les adresses des fournisseurs, emportent du matériel pédagogique, des brochures, des monographies, des idées de récompense ou de punition, scrutent les instruments destinés à observer les pensionnaires. Pendant la visite de l'établissement pour garçons de Saint-Hubert, en Belgique, les Tertiaires capucins sont assis au fond de la classe, derrière les élèves, et suivent attentivement le cours ${ }^{41}$. En 1931, deux religieux et deux religieuses sont accueillis à l'Établissement central

38 Ibid., p. 225.

39 John Boli, George M. Thomas (dir.), Constructing World Culture: International Non Governmental Organizations since 1875, Stanford, Stanford University Press, 1999; Anne Rasmussen, "Les congrès internationaux liés aux expositions universelles de Paris (1867-1900)", Mil Neuf Cent. Cahiers Georges Sorel. Revue d'histoire intellectuelle, nº7, 1989, p. 23-44. Références citées par Joëlle Droux, "L'internationalisation de la protection de l'enfance...", art. cit., p. 20.

40 Sandrine Kott, "Une "communauté épistémique” du social?», art. cit., p. 26; Christian Topalov (dir.), Laboratoires du nouveau siècle. La nébuleuse réformatrice et ses réseaux en France, 1880-1914, Paris, EHESS, 1999.

41 Adolescens, Surge!, t. 2, p. 185. 
d'observation de l'État, à Mol, en Belgique, qui a pour mission de mener des enquêtes psychopédagogiques individualisées et de développer, sur la base des résultats de l'observation, les critères nécessaires à une classification scientifique des jeunes délinquants ${ }^{42}$. Pendant leur séjour, les Tertiaires capucins sont logés à l'intérieur même de l'institution, dans des chambres mises à leur disposition, ce qui leur donne le sentiment de "faire partie de la grande famille de l'Établissement central ${ }^{43}$. Ils visitent longuement les différents bâtiments, au premier rang desquels le laboratoire de psychologie où est justement pratiquée l'observation des mineurs. Ils en retirent la conviction profonde que le personnel d'un tel établissement doit avoir reçu une formation scientifique intensive et étendue. Le directeur de la célèbre institution, Maurice Rouvroy, exerce une influence considérable sur les religieux espagnols, qui le tiennent pour "le père de la méthode d'observation "44. Il joue incontestablement un rôle de passeur ${ }^{45}$. En septembre-octobre 1934, la revue de la congrégation publie d'ailleurs intégralement une conférence que Rouvroy avait donnée lorsque les Tertiaires capucins étaient allés visiter le centre, en $1933^{46}$.

Les Tertiaires capucins organisent en tout dix voyages d'étude : deux ont lieu pendant la décennie 1900, un seul pendant les années 1910, deux pendant les années 1920 et quatre dans les années $1930^{47}$. Le premier voyage d'étude organisé par les Tertiaires capucins, qui date de 1904, n'est que peu documenté ${ }^{48}$. Cinq ans plus tard, en mai 1909, les pères Domingo de Alboraya et Javier de Valencia sont commissionnés comme on l'a vu par le gouvernement espagnol pour effectuer un voyage d'un mois : il s'agit de préparer l'ouverture de la maison de redressement de Visto Alegre, située dans les environs de Madrid. À cette occasion, ils visitent douze établissements de rééducation ou

42 David Niget, "Le genre du risque. Expertise médico-pédagogique et délinquance juvénile en Belgique au XX siècle", Histoire@Politique. Politique, culture, société, nº 14, 2011, p. 38-54.

43 Adolescens, Surge!, t. 1, p. 493.

44 Ibid., t. 1, p. 492 et 495.

45 À propos du rôle joué par Maurice Rouvroy, on verra par exemple Marie-Sylvie Dupont-Bouchat, "De la prison à l'école de bienfaisance. Origines et transformations des institutions pénitentiaires pour enfants en Belgique au XIX ${ }^{e}$ siècle (1840-1914)", Criminologie, n ${ }^{\circ}$ 1, 1995, p. 51.

46 "Disciplina y libertad (apuntes de una conferencia)", Adolescens, Surge!, t. 4, p. 243-252.

47 Tomás Roca Chust estime que le nombre réduit de voyages d'études jusqu'aux années 1930 vient du fait que "l'environnement scientifique de la congrégation n'avait pas suffisamment mûri". Tomás Roca Chust, Historia de la Congregación..., op. cit., p. 227.

48 Juan María García Latorre, "Centros de reeducación en Europa visitados por Terciarios Capuchinos (1904-1952)", ;Surgam...!, nº48, mai-juillet 2004, p. 94. 
de protection : l'un se trouve en Angleterre, un autre en France, trois en Italie et sept en Belgique. Ce voyage d'étude est le mieux connu dans la mesure où il a donné lieu à un long rapport adressé par Alboraya au ministre de l'Intérieur, Juan de la Cierva ${ }^{49}$. Le Tertiaire capucin y décrit de façon précise le fonctionnement des institutions qu'il a visitées : déroulement du séjour, critères de classification des pensionnaires, emploi du temps, organisation de l'enseignement, du travail, règlement intérieur. Alboraya indique même au ministre de l'Intérieur le montant du salaire perçu par le personnel de trois établissements de bienfaisance belges ${ }^{50}$. Le cas de l'Espagne n'est à cet égard pas isolé : en France, ou ailleurs, peu d'écoles d'infirmières ou de travail social ont été fondées sans voyage d'étude préalable aux États-Unis ou en Angleterre ${ }^{51}$. Ces missions mobilisent chaque fois une, deux ou trois religieux tout au plus, qui sont prêts à consacrer leurs vacances d'été à ces sessions d'observation. Ainsi, en 1910, un Tertiaire capucin note : "Le Père Domingo revient du voyage d'étude qui l'a mené partout en Europe pour renforcer les connaissances qu'il avait acquises lors de son voyage précédent, à l'été 1909. Il est passionné et consacre ses étés à des voyages scientifiques pour compléter la formation dispensée dans nos maisons de redressement ${ }^{52}$.

À la fin des années 1920, la congrégation religieuse répond aussi aux incitations contenues dans une ordonnance adoptée en $1926^{53}$, dans laquelle le législateur reconnaissait que la formation du personnel des maisons de redressement était insuffisante, tout en se contentant "d'encourager" le secteur privé à résoudre lui-même ce problème. Sous la dictature de Primo de Rivera (1923-1930), les prérogatives de l'Église dans le domaine éducatif sont en effet moins que jamais contestables. La décennie 1930 est sans conteste celle pendant laquelle la volonté d'ouverture et d'amélioration de la formation est la plus vive, puisque quatre voyages sont organisés. Les Tertiaires capucins cherchent alors sûrement à répondre aux critiques dont ils font l'objet : si

49 Domingo de Alboraya, Los reformatorios para jóvenes y las Colonias de beneficencia en el extranjero, Madrid, Imprenta del Asilo de Huérfanos del Sagrado Corazón de Jesús, 1910.

$50 \quad$ Ibid., p. 128.

51 Pierre-Yves Saunier, "Les régimes circulatoires...", art. cit., p. 12.

52 Mémoires du frère Lorenzo, $1^{\text {er }}$ septembre 1910. Cité par Juan María García Latorre, "Centros de reeducación en Europa...", art. cit., p. 96.

53 Real orden aprobando las normas propuestas por la Comisión directiva de los Tribunales tutelares para niños en el ejercicio de las facultades que le corresponden respecto a la autorización del funcionamiento de las instituciones auxiliares de dichos Tribunales, 14 mai 1926, Gaceta de Madrid, $\mathrm{n}^{\circ} 135,15$ mai 1926. 
celles-ci ne sont pas nouvelles, elles s'inscrivent dans un contexte politique radicalement différent. En effet, la Seconde République, proclamée en 1931, mène une politique anticléricale vigoureuse (séparation de l'Église et de l'État, interdiction faite aux congrégations religieuses d'enseigner, etc.). Les détracteurs de Santa Rita et du système rééducatif mis en place par les Tertiaires capucins réclament une épuration des établissements pour jeunes délinquants, estimant que le personnel religieux devrait être remplacé par des pédagogues, des médecins, des psychiatres et des éducateurs capables d'agir selon des critères scientifiques. Les instances qui supervisent l'action des tribunaux pour mineurs et des reformatorios sont alors le théâtre d'une lutte âpre entre une idéologie traditionnaliste et catholique, favorable aux Tertiaires capucins, et les conceptions pédagogiques progressistes de personnes qui sont souvent membres du PSOE (Parti socialiste ouvrier espagnol), de l'UGT (Union générale des travailleurs, réformiste) ou de l'ILE, comme Matilde Huici ou Luis de Zulueta. Ce dernier affirme ainsi que "désormais, avec le nouveau régime républicain, les problèmes de délinquance juvénile et de régénération de ces adolescents doivent être traités de manière plus scientifique et plus humaine $\aleph^{54}$.

Au total, les Tertiaires capucins visitent 77 institutions éducatives dans huit pays différents. Mais la Belgique, qui constitue le seul but des voyages de 1931 et de 1935, est leur destination privilégiée : les religieux se rendent à sept reprises dans ce petit État dont les réseaux réformateurs constituent, depuis la fin du XIX ${ }^{\mathrm{e}}$ siècle, de véritables laboratoires législatifs ${ }^{55}$.

\section{Voyager pour observer, apprendre et transposer}

\section{Les éléments observés}

Des divers voyages qui les mènent aux quatre coins de l'Europe occidentale pendant le premier tiers du $\mathrm{XX}^{\mathrm{e}}$ siècle, les Tertiaires capucins tirent un certain nombre de constats, attachant une importance particulière à la religion. En 1909, le père Domingo de Alboraya est favorablement impressionné par l'aspect matériel de la vie des institutions françaises qu'il visite : les terrains sur

54 Julián Palacios Sánchez, Menores marginados. Perspectiva histórica de su educación e integración social, Madrid, Editorial CCS, 1997, p. 213.

55 Marie-Sylvie Dupont-Bouchat a montré comment, depuis les années 1880-1914, la Belgique s'est érigé en terre de médiation pour les politiques transnationales. Marie-Sylvie Dupont-Bouchat, La Belgique criminelle. Droit, justice, société (XIV ${ }^{e}-X X^{e}$ siècles), Louvain-la-Neuve, Bruylant-Academia, 2006. 
lesquels celles-ci sont construites sont vastes et les bâtiments sont fonctionnels; mais il est choqué de constater que tous les établissements ne disposent pas d'une chapelle : "les pauvres enfants" ne vont pas à la messe ${ }^{56}$. Soulagés, les Tertiaires capucins visitant l'établissement pour garçons de Saint-Hubert remarquent qu'un crucifix surmonte le grand tableau noir de la salle de classe au fond de laquelle ils sont assis ${ }^{57}$. Les religieux espagnols manifestent un intérêt certain pour la colonie agricole de Mettray, qui constitue pour eux un modèle ${ }^{58}$. Ils sont également sensibles à la discipline des établissements allemands ${ }^{59}$. En 1933, quatre d'entre eux visitent le centre pour mineurs de la province de Rhénanie, situé à Mönchengladbach-Holterheide : ils notent que les pensionnaires les plus méritants paradent dans la rue, enthousiastes, en "uniforme hitlérien "60. Mais, comme on l'a vu, c'est incontestablement la Belgique qui occupe la première place du palmarès des pays visités. Les religieux ne tarissent pas d'éloge sur cet État qui est, selon le père Alboraya, "un véritable champ d'expérimentation sociale" régi par "la première législation sociale du monde ${ }^{61}$. Un peu plus de vingt ans après le voyage de 1909, la Belgique est encore considérée par les Tertiaires capucins comme "l'avant-garde des nations " ${ }^{62}$. Comme d'autres petits États, la Belgique use en effet avec habilité de son attractivité internationale pour s'imposer dans le concert des grandes puissances : elle compense sa vulnérabilité en devenant une véritable locomotive de l'internationalisme ${ }^{63}$. L'établissement d'observation de Mol constitue un

56 Domingo de Alboraya, Los reformatorios para jóvenes..., op. cit., p. 126.

57 Adolescens, Surge!, t. 2, p. 185.

58 La colonie pénitentiaire de Mettray, située dans une petite localité d'Indre-et-Loire, est destinée à réhabiliter de jeunes délinquants. Elle est créée en 1839 et ferme un siècle plus tard. L'établissement représentait au départ un modèle humaniste largement admiré; mais son image se dégrade à partir de 1880. Voir par exemple Luc Forlivesi, Georges-François Pottier, Sophie Chassat, Éduquer et punir. La colonie agricole et pénitentiaire de Mettray (1839-1937), Rennes, Presses universitaires de Rennes, 2005.

59 Adolescens, Surge!, t. 5, p. 509.

60 Ibid., p. 510.

61 Domingo de Alboraya, Los reformatorios para jóvenes..., op. cit., p. 111.

62 Adolescens, Surge!, t. 1, p. 492.

63 Sur ce rôle des petits États tels que la Belgique, voir Joëlle Droux, "L'internationalisation de la protection de l'enfance...", art. cit., p. 21 ; Sandrine Kott, "Les organisations internationales, terrains d'étude de la globalisation. Jalons pour une approche socio-historique", Critique internationale, $\mathrm{n}^{\circ} 52$, 2011, p. 15; Jasmien Van Daele, "Industrial States, Policy Preferences and International Networks: Belgium as a Case Study of a Transnational History of the ILO ", in Sandrine Kott, Joëlle Droux (dir.), Globalizing Social Rights. The International Labour Organization and beyond, New York, Palgrave, 2013. 
point de passage obligé de presque tous les voyages d'étude organisés par les Tertiaires capucins. Au total, treize religieux s'y rendent, certains plusieurs fois; il arrive même qu'ils y passent plusieurs mois. Cet établissement belge occupe une place à part dans l'imaginaire européen des Tertiaires capucins : ceux-ci s'y référent constamment et l'évoquent avec déférence et admiration. Ils louent le fait que l'enfant soit placé au cœur du dispositif éducatif, mis en place après une observation attentive de chaque individu. Les méthodes utilisées sont scientifiquement fondées, ce qui n'empêche pas l'ambiance d'être familiale $^{64}$. De fait, l'établissement d'observation de Mol jouit d'une réputation mondiale : ouvert en 1913 dans la foulée de l'adoption de la loi belge de 1912 sur la protection de l'enfance, il s'est développé jusqu'à devenir un véritable centre de recherche sur la criminalité juvénile ${ }^{65}$.

\section{Des excursions improvisées en terre étrangère}

Les récits publiés dans la revue Adolescens, Surge! montrent que la pratique des voyages d'études n'est guère enracinée dans l'habitus capucin, les religieux apparaissant comme des passagers très occasionnels des mouvements d'échanges et de transferts internationaux. Les Tertiaires capucins ne préviennent par exemple pas toujours de leur arrivée, ce qui ne manque pas d'occasionner des déconvenues. À Gand, en 1932, les religieux découvrent que l'établissement qu'ils sont venus visiter, dirigé par les frères de la Charité, a été transféré dans une autre localité66. En 1933, ils arrivent à la prison-école de Hoogstraeten, aux Pays-Bas, sans avoir demandé d'autorisation préalable au ministère : on les laisse entrer seulement parce qu'ils sont accompagnés par un collaborateur de Maurice Rouvroy, le directeur de l'établissement d'observation de $\mathrm{Mol}^{167}$. Les capucins ne sont souvent pas les seuls visiteurs, preuve que les visites d'établissements sont alors un moyen répandu de diffusion des modèles internationaux de prise en charge de l'enfance délinquante. En 1933, lorsqu'ils arrivent à Mol, les Tertiaires capucins s'intègrent à une visite en cours : le directeur est en train d'exposer le fonctionnement de l'établissement d'observation à un ministre suisse et à une Française, une demoiselle se consacrant à de bonnes

64 Adolescens, Surge!, t. 1, p. 494.

65 David Niget, "Le genre du risque...", art. cit.

66 Adolescens, Surge!, t. 2, p. 194.

67 Ibid., t. 5, p. 432. 
œuvres ${ }^{68}$. La même année, lorsque les religieux espagnols arrivent à Merxplas, les visiteurs sont déjà nombreux ${ }^{69}$.

Au cours de leurs voyages d'étude, les Tertiaires capucins sont confrontés à un problème linguistique puisqu'ils ne sont capables de s'exprimer qu'en espagnol et en français. Lorsqu'ils se rendent en Allemagne en 1933, ils doivent se faire accompagner par un collaborateur de Maurice Rouvroy, qui leur servira d'interprète ${ }^{70}$. Aux Pays-Bas, c'est le sous-directeur de la maison d'observation de La Haye qui leur fait visiter l'établissement; mais sa maîtrise du français est limitée et il ne s'exprime qu'avec difficulté ${ }^{71}$. Les récits de voyage montrent que les religieux espagnols ne dominent pas complètement la langue de Molière ${ }^{72}$. Au début de leur voyage, lorsqu'ils passent la frontière française, le 26 juin 1933, les religieux espagnols s'arrêtent à Bayonne dans un couvent tenu par des capucines, où ils s'astreignent à des exercices de traduction et de composition françaises ${ }^{73}$. Dans ce contexte, les échanges avec le personnel des établissements visités ont dû être difficiles et limités. Ces contraintes linguistiques montrent que la constitution d'un langage commun, d'un paysage familier de concepts, de manières de penser et de résoudre les problèmes ne va pas de soi : il s'agit d'un processus au cours duquel les acteurs impliqués donnent de leur temps et de leur personne. Un voyage d'étude n'est ainsi pas simplement un moment clairement délimité, mais une expérience ou une épreuve qui mobilise les êtres ${ }^{74}$.

\section{Une transposition effective des modèles?}

Il est difficile de savoir quels éléments les Tertiaires capucins ont importé en péninsule ibérique, les archives de la congrégation étant inaccessibles. Il est cependant possible de tracer quelques pistes. Tout d'abord, le nom des différents groupes de pensionnaires de la maison de redressement d'Amurrio, au Pays basque ("espérance, persévérance, confiance") vient du centre des mineurs de

68 Ibid., p. 380.

69 Ibid., p. 506.

70 Ibid., p. 508.

71 Ibid., p. 557.

72 On trouve par exemple "ll fant voir" pour "il faut voir" (Ibid., t. 2, p. 196), "la racolte» au lieu de "la récolte" (Ibid., p. 557) ou une traduction hasardeuse d'une maxime rapportée de Belgique : "Qui perséver, il arrive" (Ibid., t. 5, p. 613).

73 Ibid., t. 4, p. 282.

74 Pierre-Yves Saunier, "Les régimes circulatoires..." , art. cit. 
Saint-Hubert, en Belgique ${ }^{75}$. Par ailleurs, les apports extérieurs sont nombreux et profonds dans le domaine des appareils psychométriques. En 1929, le père José María Pérez de Alba visite l'Institut Jean-Jacques Rousseau de Genève et rencontre le professeur Édouard Claparède. Il tient également à voir le laboratoire de psychologie de l'établissement d'observation de Mol, consignant les références des appareils d'observation afin de pouvoir se les procurer, une fois rentré en Espagne $^{76}$. Enfin, les Tertiaires capucins utilisent une fiche psycho-médico-pédagogique pour dresser le bilan du niveau scolaire, religieux, de l'état de santé et psychologique des pensionnaires : ce document, qui n'évolue quasiment pas jusqu'en 1975, est une traduction presque littérale de la fiche utilisée à Mol. L'un des Tertiaires capucins ayant visité l'établissement belge en 1931 raconte la scène suivante, qui s'est déroulée dans le laboratoire psychopédagogique : "sur un grand tableau noir, sur lequel on schématise la fiche, le directeur, le médecin et les chefs des pavillons notent les résultats des tests à mesure que ceux-ci sont réalisés. Le type caractéristique de l'enfant émerge, donnant ainsi à voir le portrait complet de sa personnalité, qui permet d'établir le diagnostic et le pronostic pratique de ce cas. L'œuvre de la psychologie individuelle est terminée. Reste à accomplir celle de la pédagogie ${ }^{77}$. En 1933, les religieux notent précautionneusement les conseils délivrés par Maurice Rouvroy : "l'enfant ne doit pas être observé dans le but de remplir la fiche; celle-ci est faite pour consigner les informations nécessaires à l'éducation de l'enfant ${ }^{78}$. Les Tertiaires capucins vont jusqu'à copier les modifications successives qui sont apportées, en Belgique, au document original. Mais les religieux espagnols ajoutent une autre dimension avec l'"horloge morale" qui clôt la fiche psycho-médico-pédagogique. Cette importation directe de ressources pédagogiques fonctionne de façon horizontale, faisant fi des frontières étatiques; mais elle semble plus relever du transfert unidirectionnel que de la configuration circulatoire, au sens où l'entend Pierre-Yves Saunier ${ }^{79}$. Les Espagnols importent en effet des savoirs,

75 Notes prises à l'occasion du voyage en Belgique réalisé par Gabriel María de Ybarra y de la Revilla en 1926, archives de la congrégation. Cité par Juan María García Latorre, "Centros de reeducación en Europa...", art. cit., p. 187.

76 Notes prises par José María Pérez de Alba en 1929, ibid.

77 Adolescens, Surge!, t. 2, p. 99-100.

78 Ibid., t. 4, p. 284.

79 L'identification d'une configuration circulatoire repose notamment sur l'existence d'un groupe d'acteurs individuels et collectifs qui investissent du temps, de l'énergie et des ressources dans l'établissement de connexions destinées à faire circuler des objets spécifiques au-delà des limites de leurs communautés d'origine; sur la formation de communautés d'interconnaissances (visites, correspondance, 
des techniques et des pratiques sans nécessairement que leurs interlocuteurs n'aient été eux-mêmes demandeurs ou aient partagé les notions, les catégories ou la vision du monde des Tertiaires capucins. Le retard accumulé par l'Espagne dans le domaine de la prise en charge de la jeunesse déviante a visiblement empêché la constitution d'une communauté épistémique, entendue comme le partage d'un même savoir, d'idées et de pratiques communes ${ }^{80}$.

Après le traumatisme causé par la guerre civile espagnole (1936-1939), les Tertiaires capucins peuvent à nouveau se consacrer à leur mission première dans un contexte politique extrêmement favorable de symbiose entre le caudillo, l'Église et l'armée. La dictature franquiste (1939-1975) leur confie en effet, pour près de quarante ans, la gestion des principaux centres de rééducation tout en leur accordant "une totale liberté en matière d'éducation "81. L'Espagne se replie alors sur elle-même dans une posture autarcique qui vaut également pour les maisons de redressement et les tribunaux pour mineurs, preuve que la globalisation ne suit pas toujours un développement linéaire ${ }^{82}$. Elle se caractérise par son imperméabilité aux évolutions qui, ailleurs, se font jour : réduction de la capacité des institutions éducatives, amélioration des infrastructures, ouverture progressive des établissements vers l'extérieur, développement des relations entre les pensionnaires et leur famille, structuration du statut d'éducateur, renforcement de la formation professionnelle, diversification des activités ayant lieu pendant le temps libre, etc. Alors qu'après la Seconde Guerre mondiale, les phénomènes d'internationalisation des politiques éducatives, sanitaires et sociales s'intensifient et s'institutionnalisent (congrès de l'Association internationale des juges pour enfants, de l'Association internationale de protection de l'enfance, etc.), l'isolement autarcique de l'Espagne est tout à la fois un symptôme et une cause de l'immobilisme du dispositif de prise en charge des jeunes déviants. Dans ce contexte, il est significatif que les Tertiaires capucins n'organisent plus de voyage d'étude à l'étranger pour améliorer la formation des membres de la congrégation ${ }^{83}$. Le niveau scientifique de la prise en charge

sociabilité) et d'intertextualité (lecture, citation, traduction); sur la définition d'interactions durables entre les acteurs : Pierre-Yves Saunier, "Les régimes circulatoires...", art. cit., p. 16.

80 Sandrine Kott, "Une "communauté épistémique” du social?...", art. cit., p. 27.

81 Tomás Roca Chust, Historia de la Congregación..., op. cit., vol. 5, 1989, p. 18.

82 Sven Beckert souligne que des moments de globalisation rapide peuvent être suivis par des phases de dé-globalisation. C.A. Bayly et al., "AHR conversation: On transnational history", art. cit., p. 1460.

La guerre civile espagnole n'a pas favorisé les échanges d'expériences avec l'étranger, dans le domaine de la prise en charge de la déviance juvénile en tous cas. Pierre-Yves Saunier affirme quant à lui 
baisse notablement, tandis que les méthodes et les préceptes éducatifs utilisés n'évoluent guère. La question de l'ouverture à l'étranger et aux modèles en vigueur ailleurs fait à nouveau débat dans les années 1950, lorsque l'on tente de moderniser un système figé et archaïque. Comme elles l'avaient fait en 1909, les autorités décident de s'appuyer sur la congrégation valencienne en tentant de réactiver la configuration circulatoire qui avait fonctionné au cours du premier tiers du XX $\mathrm{XX}^{\mathrm{e}}$ siècle. Les Tertiaires capucins renouent ainsi temporairement avec la tradition des voyages d'étude à l'étranger : à l'été 1952, la revue iSurgam...! retrace le voyage d'observation ayant conduit le supérieur de la congrégation à Savigny-sur-Orge, Gand, Ruiselede, Mol, Amsterdam, Neuchâtel, etc. "Notre directeur, le père Jesús Ramos, vient de rentrer d'un voyage d'étude [...] [Il a été] commissionné par le Conseil supérieur de protection des mineurs. Ce voyage avait pour objectif de visiter les principaux établissements de tutelle et de rééducation de France, de Belgique, de Hollande, d'Allemagne de l'Ouest et de Suisse " ${ }^{84}$. Mais cette expérience n'est pas rééditée et, désormais, la congrégation religieuse d'origine valencienne ne joue plus un rôle moteur.

qu'après 1945, la guerre froide n'empêche pas la circulation des formes du savoir et des politiques du social et qu'elle peut même en être la matrice. Pierre-Yves Saunier, "Les régimes circulatoires...", art. cit., p. 14.

84 Tiré de Jesús Ramos, "Diario de un viaje", ¡Surgam...!!, nº42-43, juillet-août 1952, p. 32-36. Le Conseil supérieur de protection des mineurs supervisait l'ensemble des tribunaux pour mineurs et des maisons de redressement du pays. 